\title{
Culture and People Flow Together: Evidence from Quantitative Study of UNESCO World Heritage
}

\author{
Authors \\ Dan C. Baciu ${ }^{1}$
}

Status

Pre-print

\section{Highlights}

- Published texts about UNESCO world heritage sites reveal a burgeoning cultural exchange of global scale.

- This cultural exchange follows hierarchical pathways that connect Europe across the Atlantic to the East Coast of North and South America, and along trade routes through the Near East and India to Southeast Asia, Japan, Australia, and New Zealand.

- The pathways of cultural exchange that we observed are similar to those of air travel.

- The observation that the cultural exchange follows pathways also known from other global activities offers empirical support that culture and those other activities are indeed evolving together.

- We conclude that, on a global scale, both human thought and action follow similar hierarchical pathways.

\begin{abstract}
Culture and people flow together. While studying UNESCO world heritage sites, we found empirical evidence to support this thesis. We utilized a new method for Geographic Information Retrieval to reveal cultural connections between UNESCO world heritage sites, and between the sites and the rest of the world, and we found that these connections follow paths also beaten by other global activities, in particular air travel. The new results are relevant because they represent cultural data on heritage and preservation. In particular, UNESCO world heritage sites are a representative selection of heritage sites of universal value. The observation that cultural exchange associated with these sites follows pathways already known from other global activities offers empirical support that culture and those other activities are indeed evolving together.
\end{abstract}

\section{Keywords}

Cultural tourism; cultural exchange; air travel; contrails; constructal law.

\footnotetext{
${ }^{1}$ https://orcid.org/0000-0002-0043-5616
} 


\section{Introduction}

The United Nations Educational Scientific and Cultural Organization (UNESCO) undertakes to recognize, list, and collectively present those places around the world that are of outstanding value to all mankind and that must be protected from threats such as armed conflict, deliberate destruction, economic pressure, natural disasters, and climate change. Through the listing of such "world heritage sites," UNESCO intends to document and protect the diversity of cultures and natural phenomena around the globe and to promote international dialog.

As part of its listing, UNESCO also includes short descriptions of each site. These descriptions are a key element in the public-facing listing of the recognized world heritage sites. The texts reflect the listing in a more nuanced and elaborate form (UNESCO 2021). As a literary genre, the site descriptions may fall somewhere between encyclopedia entries and travelguide site descriptions, as they are intended both for matters of overview and as information for potential travelers.

The UNESCO world heritage list was met with much success and was welcomed by broad audiences around the globe. Given the importance of the mission to promote international dialog, we have studied the world heritage site descriptions through a new method of Geographic Information Retrieval (Baciu 2019, 2020, 2021). We were interested in exploring whether, through this new method, we can detect an international dialog, as promoted by UNESCO through the world heritage list and site descriptions. In this present essay, we discuss the results.

According to our findings, UNESCO does promote an international dialog that we are able to detect using our method. The geographical connections that we detect are very close to other known global activity. We discuss in particular the similarity between our maps of international dialog promoted by UNESCO and global movement as decoded from airplane condensation trails (contrails). Both, text and air travel, reveal similar, hierarchical connection pathways across the globe, which suggests that text and air travel (which includes people and goods) have evolved together rather than independently.

\section{World heritage listing: An evolving mission}

As of 2021, UNESCO's official website contains a list of more than thousand world heritage sites, numbered in the order in which they were included into the list. The sites are spread out across the globe, but the geographical coverage is not even. The Americas, Africa, Australia, and the northern parts of Asia show substantially less coverage than Europe. This uneven distribution may partly be due to the evolving nature of the listing.

A basic evaluation of the languages in which the listing is provided also hints at the fact that the listing is still a work in progress. The official UNESCO website includes eight languages. Italian is not among these chosen languages, although Italy claims 50 cultural sites. With this number, Italy more than doubles Russia at 18 cultural sites. In contrast to Italian, Russian is one of the eight languages in which the listing is presented. The choice of Russian over Italian language may be due to the fact that Russia is a much larger territory, and maybe UNESCO expects to have many Russian readers and more future sites in Russia. 
UNESCO recognizes both cultural and natural world heritage sites, yet, roughly $80 \%$ of the sites are cultural, while only $20 \%$ are natural. These numbers clearly set a focus on culture over nature. In addition, the percentage of cultural over natural sites is not the same across the globe. On average, Europe, Asia, and Africa have a much higher proportion of cultural sites, whereas the Americas and Australia have comparatively many natural sites. The entire West Coast of the United States has only one cultural site. Thus, we expect to see a burgeoning international dialog rather than one that is fully across all parts of the globe.

\section{Site descriptions: Brief syntheses}

The site descriptions offer the raw data for our analysis. Most descriptions begin with a brief abstract, typically less than five sentences, which is followed by a "brief synthesis" of several paragraphs. The brief syntheses are used to justify the listing of each particular site. In each synthesis, the site's most important qualities are mentioned, and potential threats are identified. This latter content sets a focus on preservation goals and preservation efforts.

The format of the descriptions is not fully formalized. For example, the title "Brief Synthesis" is sometimes capitalized, sometimes not, and sometimes, it is missing all together. These small liberties in formatting first reveled themselves to us when we processed the texts with a computer. We believe that they are healthy signs of collaboration. Many encyclopedias, including Wikipedia and SAH-Archipedia are collective efforts - the UNESCO world heritage site descriptions, too. They were contributed in a decentralized manner, involving multiple authors and editors. While this collaborative approach is often very successful, it does typically come with small liberties in formatting, as we were able to indentify.

The content of the list is "world heritage" of "universal value." This content means that the list aims to address the entire human world. The descriptions are therefore written in plain language, wherever possible. This characteristic may bring the texts closest to travel guide site descriptions. We are very familiar with such descriptions as one of us is author of such a travel guide (Baciu 2013). Thus, it can be summarized that the writing style of the texts varies across the world heritage list, but it generally falls somewhere between encyclopedia entries, catalog entries, and travel-guide site descriptions.

It is common for such texts to focus on individual objects. Therefore, when we set out to study the site descriptions, we did not know whether we would detect any international dialog. It could have been that UNESCO intended to promote international dialog, yet, the site descriptions did not reflect this effort. After evaluating the data, we can say that this hypothesis was proven wrong. The international dialog promoted by UNESCO can already be detected from the site descriptions themselves.

\section{Analysis: Combining natural language processing with geo-analysis}

The geographical evaluation that we have undertaken is aimed at assessing to which extent the UNESCO world heritage site descriptions promote a dialog between multiple sites, and between each site and the rest of the world. To achieve this goal, we have programmed a computer to decode the "geographical profile" of each site description. Our analysis is based on a new method that implements natural language processing to enrich text with geographical information (Baciu 2017, 2018, 2019, 2020, 2021). 
Technically speaking, we use the Illinois Wikifier (Ratinov et al. 2011) and data collected from Wikipedia to perform Geographic Information Retrieval GIR (Baciu 2020). Compared to previous GIR-methods, our new method allows us to identify not only unambiguous place names, but it also disambiguates ambiguous place names, recognizes alternative names, and identifies well-known institutions, businesses, infrastructures, and famous people who are mentioned in the texts. We then locate places, headquarters, birthplaces, etc. by crossreferencing our processed data to Wikipedia. Thus, the approach that we implemented is much more holistic than merely identifying toponyms, and it also has the advantage of bringing street-level resolution.

Once the UNSECO site descriptions are processed, we proceed as follows for each site:

1) We map each world heritage site as a white dot. The geolocations for this task are obtained directly from the UNESCO site descriptions.

2) We perform our GIR-method on each site description and map all content thus found as red dots. If any geo-codable item is mentioned multiple times in a given site description, we adjust the size of the given red dot to account for the total number of mentions.

3) We connect each white dot with the corresponding red dots using yellow colored gradient lines. The lines start white and end red.

4) We draw a green heat map of all dots.

Through our mapping method, we expected to be able to distinguish between two extreme situations: a highly interconnected world, and a world of disconnected islands. In a highly interconnected world, one expects to see long yellow lines that interconnect the red and white dots and frequently cross above the green heat map. In contrast, in a world of isolated islands, one does not expect to see such effects. In a world of isolated islands, one expects short yellow lines that only rarely cross. Our geographical analysis of UNESCO world heritage site descriptions reveals a highly interconnected world. We also show that our observations are not unique; our map obtained by studying text is very similar to maps obtained by studying air travel.

\section{Results: International dialog close to connectivity known from air travel}

Figure 1 shows a static version of our mapping of UNESCO world heritage site descriptions. The interactive version can be accessed through the link given in the image caption. The visual shows that the UNESCO world heritage sites are richly interconnected especially across the Atlantic. Strong connections are seen between Europe and the East Coast of North America and between Europe and Central and South America. Many, although shorter-range connections also exist along trade routes from Europe through the Near East and India to Southeast Asia, China, and Japan, as well as Australia and New Zealand. A closer look also reveals that Southeast Asia is within itself highly interconnected (Thailand, Singapore, Indonesia, Vietnam, Philippines). Connections across the pacific are rare. 


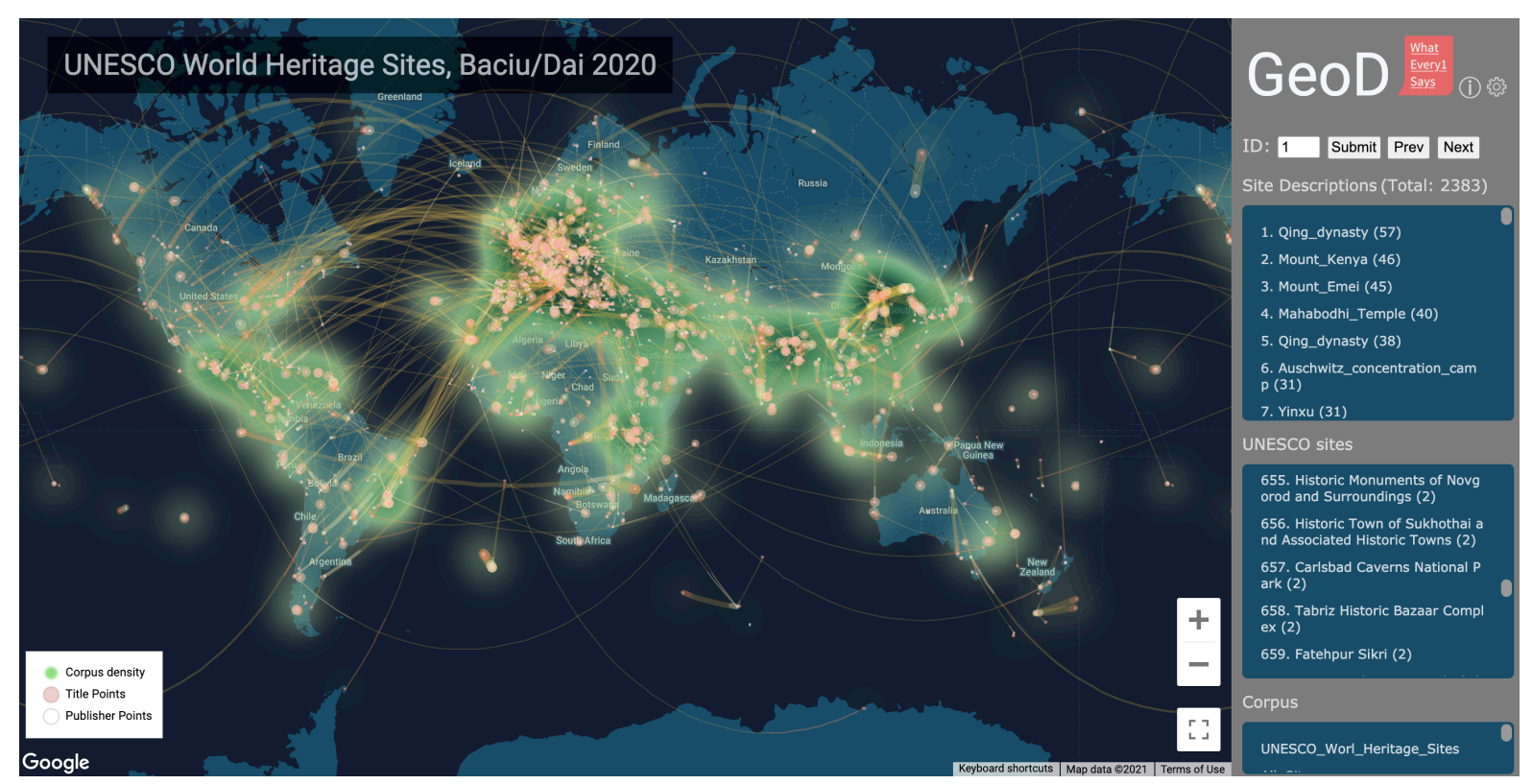

Fig. 1. International discourse supported by the UNESCO world heritage site descriptions and decoded through our method (Baciu 2020). https://unesco-whs.herokuapp.com

The question, which areas are highly interconnected, and which not, is a difficult one. One could imagine countless different ways to interconnect the listed world heritage sites between themselves and with the rest of the world. The multitude of such possibilities can be illustrated for example by calculating how many ways there are to arbitrarily draw 1'000 lines between 1'000 world heritage sites. There are billions of different possible outcomes, and the more lines are drawn, the more possibilities there are. To understand what we see, let us therefore search for a close match to our observations. Are there other maps of global activity that come close to the discourse created by the UNESCO world heritage listing? We have already suggested that the world heritage site descriptions come close to travel-guide site descriptions. What if we look at global air travel; does our map resemble global air travel pathways and distributions (Fig. 2)?

The answer is yes. In Figure 2, a static version of global air travel is displayed. An interactive version can be accessed through the link given in the image caption. The map on display is obtained through satellite images that record the white condensation trails (contrails) left behind jet airplanes. The contrails include passenger and freight flights.

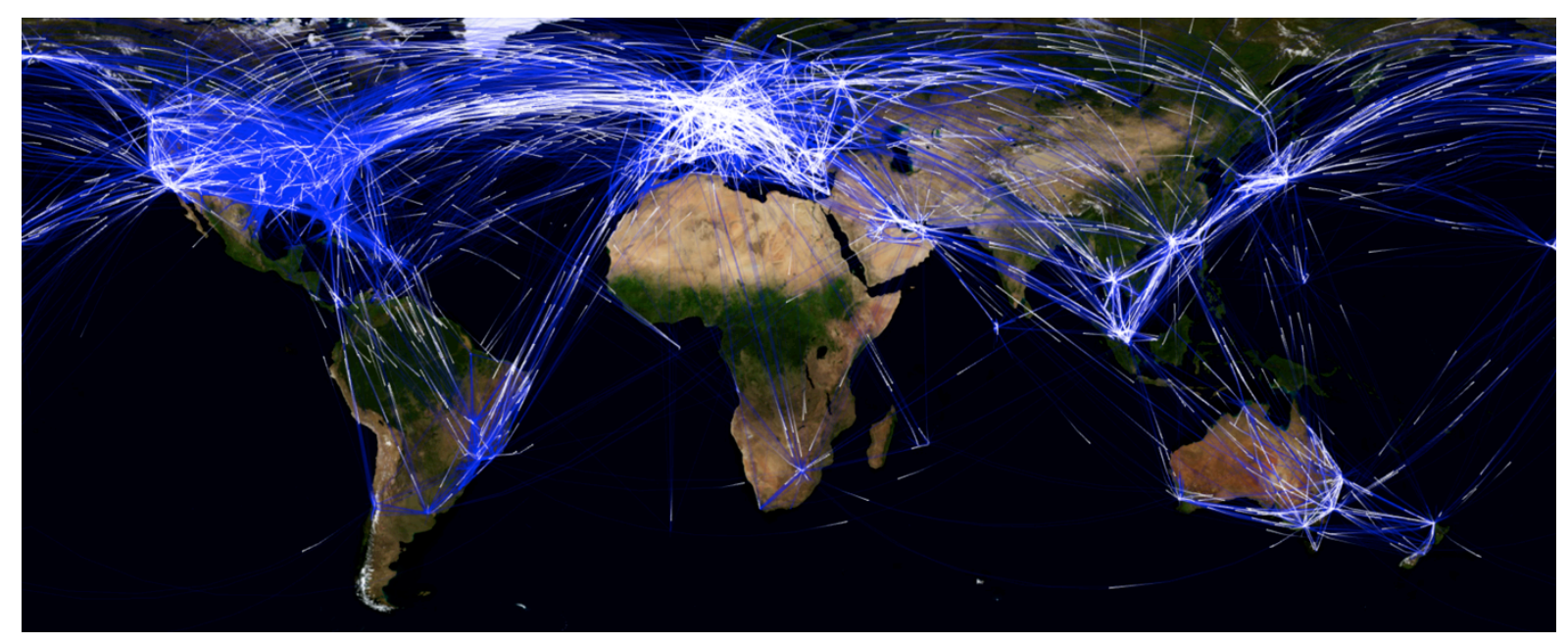


Fig. 2. International connectivity as recorded by observing airplane condensation trails (West 2012). The maps shows a similar distribution, pathways, and hierarchy thereof, as our map of international discourse decoded from UNESCO world heritage site descriptions.

Interactive at https://contrailscience.com/map/map.php

Both the international discourse decodable from the UNESCO world heritage site descriptions and the map of global air travel display the same hierarchical architecture. The connection across the Atlantic from Europe to North America is the strongest, followed by other connections between Europe and South America and along trade routes from Europe through the Near East and India to Southeast Asia, China, and Japan, as well as Australia and New Zealand, as described above. Not previously observed on our map is a strong connection between the West Coast of North America and Asia.

The hierarchical nature of these connection-pathways is a natural phenomenon, predictable through the study of flow systems (constructal law, Bejan 2012). It is natural that some pathways are much larger than others. What may be most interesting, is that our map and this map of air travel show the same picture, overall. This means that the two maps may have evolved together. The international discourse supported by UNESCO is not an independent phenomenon. It is part of global human thought and activity, in general.

The probably biggest difference between the two maps is how the data are sampled. The map of global air travel is drawn based on images collected from thousands of satellites that are quite evenly distributed over the entire globe. The distribution of satellites is shown in Figure 3. On the other hand, the UNESCO are not evenly distributed over the globe, as mentioned in section 2 and illustrated in Figure 4. World heritage sites are a representative collection of world heritage, yet, they can presently only make up for a much smaller and less even sampling that that gained from satellite images. We only look at roughly thousand heritage sites in total, each represented with only one short descriptive text, and the distribution on the globe is uneven. This means that the sample size is much smaller and fluctuations are expected to be higher. This phenomenon is best seen over the Pacific. The West Coast of the United States is not well covered by UNESCO, and in consequence, the connections between the North American West Coast and East Asia are almost absent. The coverage is spotty. In this respect, it may be interesting to think about possibilities of closing the loop and strengthening connections across the Pacific.

Finally, we should stress that the he international dialog that we have detected is constructed only for the study of world heritage site descriptions. In contrast, UNESCO's effort to promote international dialog may be much boarder and more varied, and the world heritage list may have been successful in many other ways that we would like to research on at a later stage. It is all the more remarkable that, already on this basis, UNESCO does support international dialog, and one that is close to the way people actually travel around the globe. 


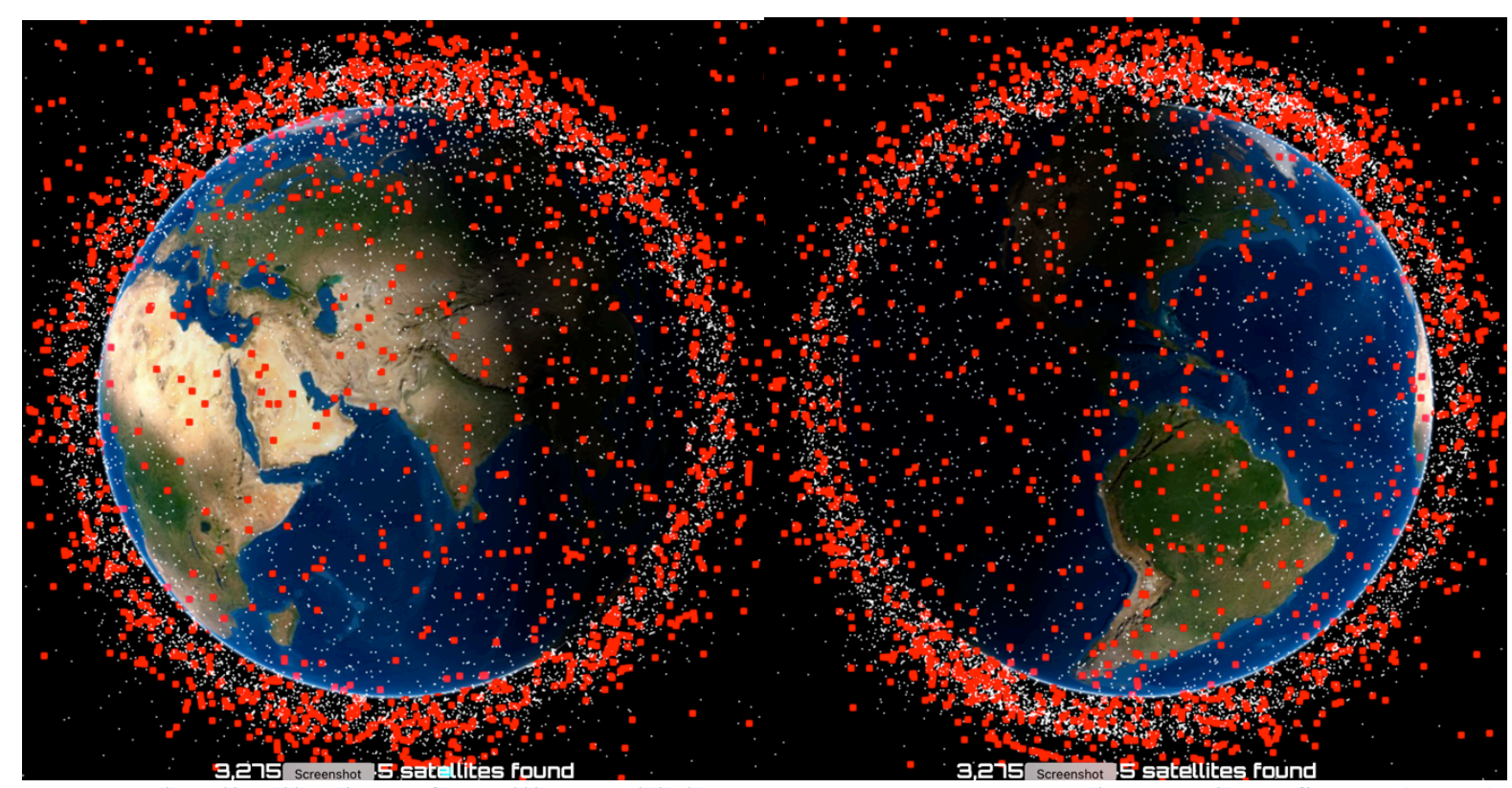

Fig. 3. The distribution of satellites, which are necessary to create the previous figure (2012). Interactive at https://maps.esri.com/rc/sat2/index.html

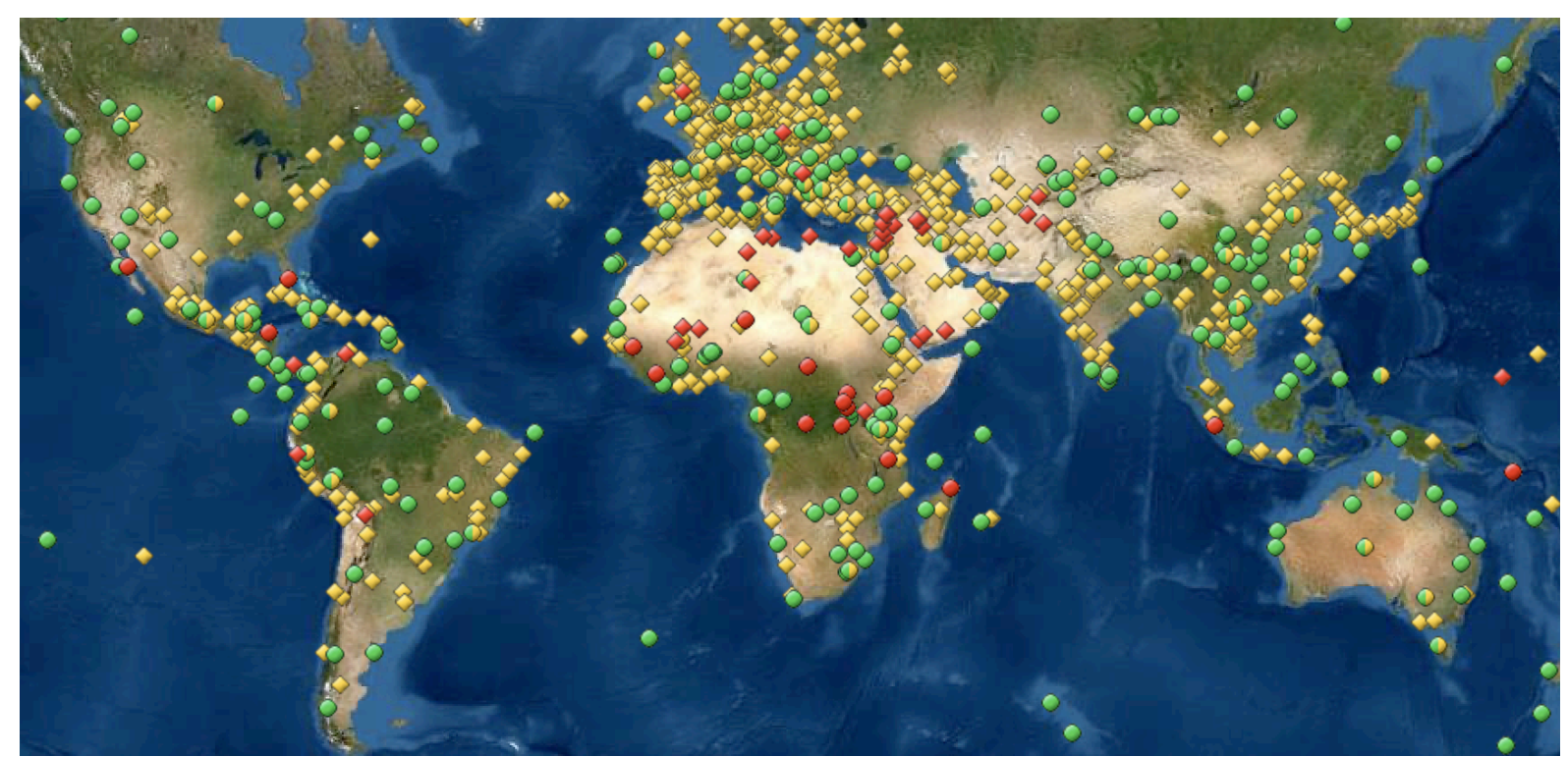

Fig. 4. The distribution UNESCO world heritage sites around the globe. They are the starting point of our analysis. Compared to satellites, the UNESCO data is sparser and the distribution is less even, with little coverage for example in North America. Hence, we expect some connections to be less well visible. This goes in particular for the connection between East Asia and North America, which will show only faintly in our analysis because of the uneven UNESCO world heritage site coverage. It may be worth thinking about ways to strengthen this trans-Pacific connection and close the loop of international dialog.

Interactive at https://whc.unesco.org/en/list/ 


\section{Discussion}

The UNESCO World Heritage list has been immensely successful in many respects. It found broad audience and public interest, and it certainly promoted international exchange between diverse people and their cultures. Such promotion may have happened through the making of the list itself, or through the bringing together of people from diverse backgrounds onto heritage sites of universal value. Here, we showed that the world heritage listing, and in particular the world heritage site descriptions already document an international dialog. This dialog stretches across the globe along paths already used by people and goods that travel, which suggests that global travel and global cultural exchange have evolved together, rather than separately. Finally, we conclude that the pathways for international exchange, which we have observed, are deeply rooted in human culture and society and are relevant for understanding how the built and natural environment is transformed through both human action and thought.

\section{Acknowledgments}

We would like to acknowledge the work of Tianchen Dai ${ }^{2}$, who has collected the UNESCO data from the internet and run the preprocessing.

\section{Conflict of Interest}

The authors declare no conflict of interest.

\section{Bibliography}

Baciu DC (2013). Die Schonsten Bauten 1960-75; Les Plus Beaux Batiments 1960-75. Patrimoine Suisse, 2013.

Baciu DC (2017). “The Chicago School: Evolving Systems of Value.” Report, HathiTrust Research Center.

Baciu DC (2018). "From Everything Called Chicago School Towards the Theory of Varieties." Doctoral dissertation, Illinois Institute of Technology.

Baciu DC (2019). "The Chicago School: Large-Scale Dissemination and Reception.” Prometheus 2, 20-41.

Baciu DC (2020). "Cultural Life: Theory and Empirical Testing." Biosystems 197.104208. DOI: https://doi.org/10.1016/j.biosystems.2020.104208

Baciu DC (2021). “Is “Cultural Life” a Form of Life?” Lecture, TU Delft, published in: Igloo Feb. 2021, 58-61.

Bejan A (2012). "Why we want power: Economics is physics." International Journal of Energy and Mass Transfer 05.046. DOI: https://doi.org/10.1016/j.ijheatmasstransfer.2012.05.046

\footnotetext{
${ }^{2}$ https://orcid.org/0000-0002-8050-8239
} 
Ratinov L, D Roth, D Downey, M Anderson (2011). "Local and Global Algorithms for Disambiguation to Wikipedia." $A C L$.

UNESCO (2021). "World Heritage List." Whc.unesco.org, retrieved April 12. 2021 from https://whc.unesco.org/en/list/

West M (2012). "Interactive Flight/Contrail Map Visualization.” contrailscience.com, retrieved April 12. 2021 from https://contrailscience.com/interactive-flight-mapvisualization/ 